\title{
Analytical Solution of Magnetic Field in Permanent-Magnet Eddy-Current Couplings by Considering the Effects of Slots and Iron-Core Protrusions
}

\author{
Xin Dai, Qinghua Liang, Chao Ren, Jiayong Cao, Jinqiu Mo, and Shigang Wang* \\ The State Key Laboratory of Mechanical System and Vibration, School of Mechanical Engineering, \\ Shanghai Jiao Tong University, 800 Dongchuan Road, Shanghai 200240, China
}

(Received 20 April 2015, Received in final form 20 June 2015, Accepted 25 June 2015)

\begin{abstract}
In this study, we propose an analytical model for studying magnetic fields in radial-flux permanent-magnet eddy-current couplings by considering the effects of slots and iron-core protrusions on the eddy currents. We focus on the analytical prediction of the air-gap field by considering the influence of eddy currents induced in conducting bars. In the proposed model, the permanent magnet region is treated as the source of a timevarying magnetic field and the moving-conductor eddy current problem is solved based on the resolution of time-harmonic Helmholtz equations. The spatial harmonics in the air gap and in slots, as well as the time harmonics are all considered in the analytical calculation. Based on the proposed field model, the electromagnetic torque is computed by using the Maxwell stress tensor method. Nonlinear finite element analysis is performed to validate the analytical model. The proposed model can be used for permanent-magnet eddy-current couplings with any slot-pole combination.
\end{abstract}

Keywords : eddy current coupling, iron-core protrusion, permanent magnet, slotting effect, subdomain model

\section{Introduction}

Permanent magnet (PM) eddy-current coupling (PMEC) is a useful device for power transmission and speed adjustment, which has been studied intensively in recent years [1-6]. In general, the conductor rotor is slotless in PMEC. In order to enhance the capacity for power transmission, a slotted conductor rotor topology with the axialflux type was proposed in [1], which can substantially improve the torque density of PMEC.

The study of the magnetic field in PM devices can be achieved in two ways: numerical methods [1,2] and analytical methods [3-12]. Numerical methods such as the finite element (FE) method can yield accurate predictions of the performance of a device by considering the real geometry and nonlinear material properties, but the $\mathrm{FE}$ method is time-consuming because accurate results require a refined mesh of the model, which may limit its application to design optimization. By contrast, analytical methods require much less computational resources and computational time, and they can be effective for making

(C)The Korean Magnetics Society. All rights reserved.

*Corresponding author: Tel: +86-21-3420-4496

Fax: +86-21-3420-4496, e-mail: wangshigang@sjtu.edu.cn rapid evaluations of the performance of PMEC with specific settings for the design parameters.

The layer model method is used widely as an analytical method for slotless PM devices [3-7]. This method divides the model into several layers where it assumes that the physical properties in each layer are homogeneous. However, this method is not applicable to the slotted model due to the slots and iron-core protrusions, which lead to discontinuity in the conductor layer. Another method that has been used recently is the magnetic equivalent circuit (MEC) method [8]. Complex geometry and nonlinear material properties can be considered with the MEC method, whereas they are normally simplified or ignored in analytical models. However, when employing this method, it is necessary to know the magnetic path before analytical modeling. Moreover, for a model where the air gap is large or variable in width, such as the PMEC, the field predictions obtained using this method lack accuracy. The subdomain method is used frequently for models with slotted rotor topology such as PM machines [9-13]. This method divides the discontinuous field into several simple solution domains and the field distributions can then be obtained by solving the governing equations in each subdomain by applying the boundary conditions. In the study of PM machines based on the subdomain model, 


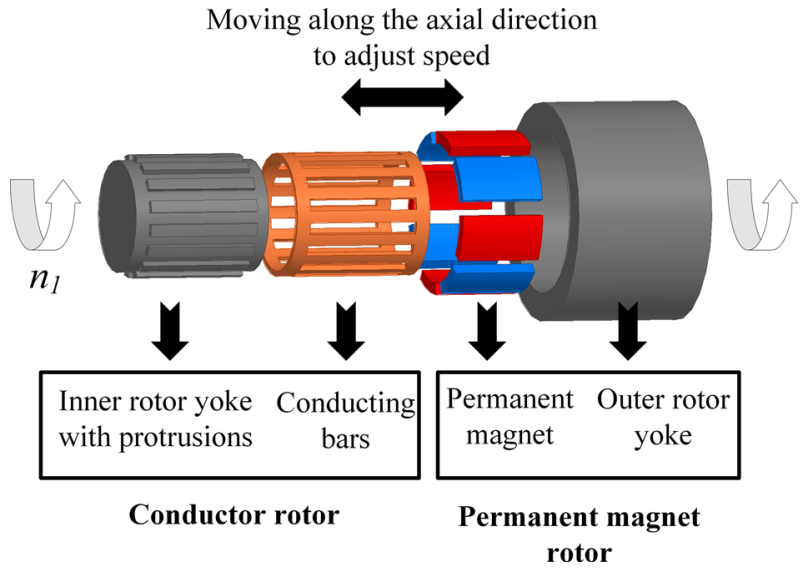

Fig. 1. (Color online) Slotted PMEC with iron-core protrusions.

previous studies have often focused on predicting the airgap field distributions without considering the influence of eddy currents $[9,10]$, or predicting the eddy current loss in magnets [11, 12], windings [9, 10], and cores [13], where the topologies are isolated with no mutual influence, and thus their solutions are not applicable to the modeling of the slotted conductor rotor shown in Fig. 1.

The torque performance and the parameter analysis were investigated to some extent in [1] using the FE method, but predicting the magnetic field using an analytical method has not been reported previously, although this would be of great utility in preliminary design work and parameter optimization. In the present study, we propose an accurate analytical subdomain model that considers the effects of slots and iron-core protrusions to predict the magnetic field distribution of a radial-flux PMEC. In this derivation, we consider the effects of eddy currents induced in the conducting bars on the air-gap field, the mutual effects between the conducting bars, and the effects of the spatial harmonics of slots. The PM region is treated as the source of a time-varying magnetic field and the moving-conductor eddy-current problem is solved based on the resolution of time-harmonic Helmholtz equations. The FE method results verified the validity of the analytical model.

\section{Analytical Field Modeling}

\subsection{Geometry and assumptions}

The geometric representation of the model used to derive the air-gap magnetic field of a radial-flux PMEC is shown in Fig. 2. The geometric parameters comprise the inner radius of the yoke of the conductor rotor $R_{\mathrm{ci}}$, the radius of the conductor rotor surface $R_{\mathrm{co}}$, the inner radius of the magnets $R_{\mathrm{mi}}$, and the outer radius of the magnets

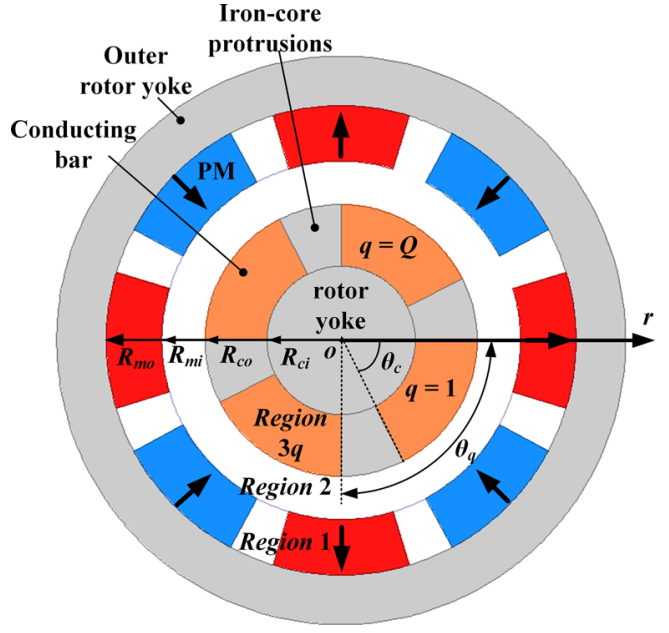

Fig. 2. (Color online) Symbols and geometries of the subdomain model investigated in this study.

$R_{\mathrm{mo}}$. The number of pole pairs is $p$ and the pole-arc to pole-pitch ratio is $\alpha$. The conductor rotor has $Q$ slots and the slot opening angle is $\theta_{c}=2 \pi \beta / Q$, where $\beta$ is the bararc to slot-pitch ratio. The initial position of the $q$-th spoke is defined as $\theta_{q}=2 \pi(q-1) / Q$.

In the derivation, as shown in Fig. 2, the two-dimensional (2D) analytical model is divided into three types of subdomains, i.e., PM (region 1), air gap (region 2), and conducting bars (region 3). The analytical modeling process is based on the following assumptions, which are commonly employed in the analysis of PM devices: (1) the permeability of the yoke and protrusions is infinite; (2) the conductivity of the yoke is neglected; (3) the axial length of the conducting bars and the magnets is infinite, and the end effect along the axial direction is neglected; (4) the magnetic vector potential $A$ in each sub-region has only an axial component; (5) the conducting bars are shorted to each other through end rings; (6) the value of the permeability of the air, bars, and magnets is 1 ; and (7) the magnets are radial magnetized.

Moreover, in order to simplify the theoretical analysis of the eddy current problem in the sub-regions of the conducting bars, then based on a previous study [4], the PM region is treated as the source of a time-varying magnetic field. In the polar coordinates, the magnetization distribution of the PM in region 1 along the circumferential direction can be expanded into a Fourier series

$$
M_{r}=\sum_{m} M_{m} \cos \left[m p\left(\theta-\theta_{\delta}\right)\right]
$$

where

$$
M_{m}=\frac{4 B_{r} p}{\mu_{0} \lambda_{p m, m} \pi} \sin \left(\frac{\lambda_{p m, m} \pi \alpha}{2 p}\right)
$$




$$
\lambda_{p m, m}=m p \text { with } m=1,3,5, \ldots
$$

and where $\theta_{\delta}$ is the initial angle position of the PM rotor, $r$ and $\theta$ are the radial and circumferential positions, respectively, and $B_{r}$ is the remanence of the magnet.

By considering the time variable, the magnetization in complex notation can be written as

$$
\tilde{M}_{r, t}(r, \theta, t)=\sum_{m} M_{m} e^{j \Delta \omega t-j \lambda_{p m, m}\left(\theta-\theta_{s}\right)}=\sum_{m} \tilde{M}_{m}(r, \theta) e^{j \Delta \Delta t},
$$

where $\Delta \omega$ is the angular frequency:

$$
\Delta \omega=n_{1} s \frac{2 \pi}{60} \lambda_{p m, m},
$$

and where $n_{1}$ is the input speed of the primary, $s$ is the slip, and $m$ is the order of the time harmonic. A tilde over a variable denotes its complex form, containing the complex quantity $j=\sqrt{-1}$.

It should be noted that the vector potentials in each subregion vary sinusoidally with time at the same regular frequency $\Delta \omega$. Therefore, they can be expressed in complex notation as

$$
A(r, \theta, t)=\operatorname{Re}[\tilde{A}(r, \theta, t)] \boldsymbol{e}_{z}=\operatorname{Re}\left[\tilde{A}_{i}(r, \theta) e^{j \Delta \omega t}\right] \boldsymbol{e}_{z},
$$

where $\boldsymbol{e}_{\mathrm{z}}$ is the unit vector in the axial direction and $i$ refers to the order of the regions.

\subsection{Vector potential distributions}

It is known that the magnetic field in each region can be expressed by the vector potential as

$$
\nabla \times(\nabla \times \boldsymbol{A})=\mu_{0}(\nabla \times \boldsymbol{H}+\nabla \times \boldsymbol{M}),
$$

where $\boldsymbol{A}$ is the vector potential, $\boldsymbol{H}$ is the field intensity, and $\boldsymbol{M}$ is the magnetization.

Therefore, at any time in region 1 , the field equation is

$$
\frac{\partial^{2} \tilde{A}_{1, m, t}}{\partial r^{2}}+\frac{1}{r} \frac{\partial \tilde{A}_{1, m, t}}{\partial r}+\frac{1}{r^{2}} \frac{\partial^{2} \tilde{A}_{1, m, t}}{\partial \theta^{2}}=\frac{\mu_{0}}{r} \frac{\partial \tilde{M}_{r, m, t}}{\partial \theta} .
$$

According to assumption (1), we have the boundary condition

$$
\left.\frac{\partial \tilde{A}_{1, m, t}}{\partial r}\right|_{r=R_{m o}}=0 .
$$

Then the general solution of (8) can be given by

$$
\tilde{A}_{1, m}=\tilde{A}_{1}+\sum_{n=1}^{\infty}\left[\begin{array}{cc}
\tilde{B}_{1} R_{m o}^{n} U_{r, R_{m o}}^{(n)} & \cos (n \theta) \\
+\tilde{C}_{1} R_{m o}^{n} U_{r, R_{m o}}^{(n)} & \sin (n \theta)
\end{array}\right]+\tilde{A}_{p},
$$

where $\tilde{A_{1}}, \tilde{B_{1}}$, and $\tilde{C_{1}}$ are the constant coefficients that need to be determined, and the following notation is adopted [10]

$$
U_{x, y}^{(z)}=\left(\frac{x}{y}\right)^{z}+\left(\frac{y}{x}\right)^{z} .
$$

The expressions for the particular solution $\tilde{A}_{p}$ in (10) are: if $\lambda_{p m, m} \neq 1$, then

$$
\begin{aligned}
\tilde{A}_{p}= & {\left[\left(\frac{R_{m o}}{r}\right)^{n} \frac{R_{m o}}{n} \tilde{M}_{m}^{(1)}+r \tilde{M}_{m}^{(1)}\right] \cos \left(\lambda_{p m, m} \theta\right) } \\
& +\left[\left(\frac{R_{m o}}{r}\right)^{n} \frac{R_{m o}}{n} \tilde{M}_{m}^{(2)}+r \tilde{M}_{m}^{(2)}\right] \sin \left(\lambda_{p m, m} \theta\right)
\end{aligned}
$$

and if $\lambda_{p m, m}=1$, then

$$
\begin{aligned}
\tilde{A}_{p}= & {\left[-\left(\frac{R_{m o}}{r}\right)^{n} \frac{R_{m o}}{n}\left(\ln R_{m o}+1\right) \tilde{N}_{1}-r \ln r \tilde{N}_{1}\right] \cos \theta } \\
& +\left[-\left(\frac{R_{m o}}{r}\right)^{n} \frac{R_{m o}}{n}\left(\ln R_{m o}+1\right) \tilde{N}_{2}-r \ln r \tilde{N}_{2}\right] \sin \theta
\end{aligned}
$$

where

$$
\begin{aligned}
& \tilde{M}_{m}^{(1)}=\frac{j 4 B_{r} p}{\pi\left(\lambda_{p m, m}^{2}-1\right)} \sin \left(\frac{\lambda_{p m, m} \pi \alpha}{2 p}\right) e^{j \lambda_{p m, m} \theta_{\delta}} \\
& \tilde{M}_{m}^{(2)}=\frac{4 B_{r} p}{\pi\left(\lambda_{p m, m}^{2}-1\right)} \sin \left(\frac{\lambda_{p m, m} \pi \alpha}{2 p}\right) e^{j \lambda_{p m, m} \theta_{\delta}} \\
& \tilde{N}_{1}=\frac{2 j B_{r}}{\pi} \sin \left(\frac{\pi \alpha}{2}\right) e^{j \theta_{\delta}} \\
& \tilde{N}_{2}=\frac{2 B_{r}}{\pi} \sin \left(\frac{\pi \alpha}{2}\right) e^{j \theta_{\delta} .}
\end{aligned}
$$

According to the assumption, the source of the magnetic field is time-varying. Based on (6), the eddy current density induced in region $3 q$ can be derived as

$$
\tilde{J}_{3 q, m, t}=-\sigma \frac{\partial \tilde{A}_{3 q, m, t}}{\partial t} .
$$

Thus, the governing function for region $3 q$ is a Helmholtz equation, which can be concluded as

$$
\frac{\partial^{2} \tilde{A}_{3 q, m, t}}{\partial r^{2}}+\frac{1}{r} \frac{\partial \tilde{A}_{3 q, m, t}}{\partial r}+\frac{1}{r^{2}} \frac{\partial^{2} \tilde{A}_{3 q, m, t}}{\partial \theta^{2}}=j \mu_{0} \sigma \Delta \omega \tilde{A}_{3 q, m, t} .
$$

Considering the infinite permeability of the iron-core protrusion, the boundary conditions on the surface of protrusions are

$$
\left.\frac{\partial \tilde{A}_{3 q, m, t}}{\partial \theta}\right|_{\theta=\theta_{q}}=0 \text { and }\left.\frac{\partial \tilde{A}_{3 q, m, t}}{\partial \theta}\right|_{\theta=\theta_{q}+\theta_{c}}=0,
$$

which is a Sturm-Liouville problem with homogeneous Neumann boundary conditions. In addition, applying the 
boundary condition of the inner rotor yoke surface which requires

$$
\left.\frac{\partial \tilde{A}_{3 q, m, t}}{\partial \theta}\right|_{r=R_{c i}}=0
$$

and the general solution of vector field in region $3 q$ can be obtained as

$$
\begin{aligned}
\tilde{A}_{3 q, m}= & \tilde{A}_{3 q}\left[J_{0}(\rho r)-\tilde{\Omega}_{1} Y_{0}(\rho r)\right] \\
& +\sum_{k=1}^{\infty} \tilde{C}_{3 q}\left[J_{\lambda_{b, k},}(\rho r)-\tilde{\Omega}_{k} Y_{\lambda_{b, k},}(\rho r)\right] \cos \left[\lambda_{c b, k}\left(\theta-\theta_{q}\right)\right]
\end{aligned}
$$

where $\tilde{A}_{3 q}$ and $\tilde{C}_{3 q}$ are the constant coefficients that need to be determined, $k$ is the order of the spatial harmonic caused by the spatial distribution of bars, $J_{x}$ is the Bessel function with order $x$, and $Y_{x}$ is the Neumann function with order $x$ :

$$
\begin{aligned}
& \lambda_{c b, k}=\frac{k \pi}{\theta_{c}} \\
& \rho^{2}=-j \mu_{0} \sigma \Delta \omega \\
& \tilde{\Omega}_{1}=\frac{J_{1}\left(\rho R_{c i}\right)}{Y_{1}\left(\rho R_{c i}\right)} \\
& \tilde{\Omega}_{k}=\frac{\lambda_{c b, k} J_{\lambda_{b b, k}}\left(\rho R_{c i}\right)-R_{c i} \rho J_{\lambda_{c b, k}+1}\left(\rho R_{c i}\right)}{\lambda_{c b, k} Y_{\lambda_{c b, k}}\left(\rho R_{c i}\right)-R_{c i} \rho Y_{\lambda_{c i b k}+1}\left(\rho R_{c i}\right)} .
\end{aligned}
$$

It should be noted that, unlike the analytical model used for predicting the eddy currents density in PM machines [9-13], for the model proposed in the present study, the eddy currents flow between the bars via the axial end ring, and thus the interaction between the bars must be considered. According to (18) and (22), the sum of the eddy currents induced in region $3 q$ in terms of the $m$-th time harmonic is

$$
\tilde{I}_{3 q, m, t}=\int_{R_{c i}}^{R_{c o}} \int_{\theta_{q}}^{\theta_{q}+\theta_{c}}-j \sigma \Delta \omega A_{3 q, m} e^{j \Delta \omega t} d \theta d r .
$$

The sum of the currents in all of the conducting bars is zero, i.e.,

$$
\sum_{q=1}^{Q} \tilde{I}_{3 q, m, t}=0
$$

so (28) can be developed further as

$$
\sum_{q=1}^{Q} \tilde{A}_{0}^{3 q}=0 .
$$

Equation (29) indicates the relations between the magnetic quantities in the conducting bars.

The governing function for region 2 is

$$
\frac{\partial^{2} \tilde{A}_{2, m, t}}{\partial r^{2}}+\frac{1}{r} \frac{\partial \tilde{A}_{2, m, t}}{\partial r}+\frac{1}{r^{2}} \frac{\partial^{2} \tilde{A}_{2, m, t}}{\partial \theta^{2}}=0 \text {. }
$$

The general solution of the vector field in this region is

$$
\tilde{A}_{2, m}=\tilde{A}_{2}+\tilde{B}_{2} \ln r+\sum_{n=1}^{\infty}\left[\begin{array}{l}
\left(\tilde{C}_{2} r^{n}+\tilde{D}_{2} r^{-n}\right) \cos (n \theta) \\
+\left(\tilde{E}_{2} r^{n}+\tilde{F}_{2} r^{-n}\right) \sin (n \theta)
\end{array}\right],
$$

where $\tilde{A}_{2}, \tilde{B_{2}}, \tilde{C_{2}}, \tilde{D_{2}}, \tilde{E}_{2}$, and $\tilde{F}_{2}$ are the constant coefficients that need to be determined, and $n$ is the order of the spatial harmonic in regions 1 and 2 .

\subsection{Interface conditions}

It can be seen that the expressions for the vector potential distributions in regions 1 and 3 already consider the boundary conditions along the surfaces of the inner/ outer rotor yoke and protrusions. Thus, in order to investigate the effects of the eddy current reaction field, the interface conditions between regions should be considered, thereby establishing a strong-coupling model to study the influence of the eddy currents on the air-gap field and PM region.

A. Interface between regions 1 and 2

The vector potential is continuous between regions 1 and 2 , which requires

$$
\tilde{A}_{1, m, t}\left(R_{m i}, \theta\right)=\tilde{A}_{2, m, t}\left(R_{m i}, \theta\right) .
$$

By applying the interface condition, the expression for the vector potential in region 1 can be developed as

$$
\begin{aligned}
\tilde{A}_{1, m}=\tilde{A}_{0}^{(1)}+\sum_{n=1}^{\infty}\left\{\begin{array}{l}
{\left[A_{n}^{(1)} \frac{U_{r, R_{m o}}^{(n)}}{U_{R_{m m}, R_{m o}}^{(n)}}+\eta_{m 1} \tilde{M}_{m}^{(1)} S_{n, r}+\eta_{m 2} \tilde{N}_{1} \xi_{r}\right] \cos (n \theta)} \\
+\left[C_{n}^{(1)} \frac{U_{r, R_{m o}}^{(n)}}{U_{R_{m i}, R_{m o}}^{(n)}}+\eta_{m 1} \tilde{M}_{m}^{(2)} S_{n, r}+\eta_{m 2} \tilde{N}_{2} \xi_{r}\right] \sin (n \theta)
\end{array}\right\} \\
S_{n, r}=-\left(\frac{R_{m o}}{R_{m i}}\right)^{n} \frac{R_{m o}}{n} \frac{U_{r, R_{m o}}^{(n)}}{U_{R_{m i}, R_{m o}}^{(n)}}-R_{m i} \frac{U_{r, R_{m o}}^{(n)}}{U_{R_{m i}, R_{m o}}^{(n)}}+\left(\frac{R_{m o}}{r}\right)^{n} \frac{R_{m o}}{n}+r \\
\xi_{r}=R_{m o} \frac{R_{m o}}{R_{m i}} \frac{U_{r, R_{m o}}^{(1)}}{U_{R_{m i}}^{(1)}, R_{m o}}\left(\ln R_{m o}+1\right)+R_{m i} \ln R_{m i} \frac{U_{r, m_{m o}}^{(1)}}{U_{R_{m i}, R_{m o}}^{(1)}} \\
-R_{m o} \frac{R_{m o}}{r}\left(\ln R_{m o}+1\right)-r \ln r,
\end{aligned}
$$

where $\tilde{A}_{0}^{(1)}, \tilde{A}_{\mathrm{n}}^{(1)}$ and $\tilde{C}_{\mathrm{n}}^{(1)}$ are the constant coefficients that need to be determined. $\eta_{m 1}$ and $\eta_{m 2}$ lead to:

$$
\begin{aligned}
& \text { if } \lambda_{p m, m} \neq 1, \text { then } \\
& \eta_{m 1}=1 \text { and } \eta_{m 2}=0 ;
\end{aligned}
$$

and if $\lambda_{p m, m}=n=1$, then

$$
\eta_{m 1}=0 \text { and } \eta_{m 2}=1 ;
$$

otherwise,

$$
\eta_{m 1}=\eta_{m 2}=0 .
$$


For the undetermined coefficients, the subscript denotes the number of the region and the subscript denotes the harmonic order. The coefficients in (33) can be determined by

$$
\begin{aligned}
\tilde{A}_{n}^{(1)} & =\frac{1}{\pi} \int_{0}^{2 \pi} \tilde{A}_{2, m}\left(R_{m i}, \theta\right) \cos (n \theta) d \theta \\
& =M_{n \times n}^{(1)} \tilde{A}_{n}^{(2)}-M_{n \times n}^{(2)} \tilde{B}_{n}^{(2)} \\
\tilde{C}_{n}^{(1)} & =\frac{1}{\pi} \int_{0}^{2 \pi} \tilde{A}_{2, m}\left(R_{m i}, \theta\right) \sin (n \theta) d \theta . \\
& =M_{n \times n}^{(1)} \tilde{C}_{n}^{(2)}-M_{n \times n}^{(2)} \tilde{D}_{n}^{(2)}
\end{aligned}
$$

B. Interface between regions 2 and $3 q$

At the interface between regions $3 q$ and 2 , the vector potential is continuous, which requires

$$
\tilde{A}_{2, m, t}\left(R_{c o}, \theta\right)=\tilde{A}_{3 q, m, t}\left(R_{c o}, \theta\right) \text {. }
$$

By applying (41), expression (22) can be developed as

$$
\begin{aligned}
\tilde{A}_{3 q, m}= & \tilde{A}_{0}^{(3 q)} \frac{J_{0}(\rho r)-\tilde{\Omega}_{1} Y_{0}(\rho r)}{J_{0}\left(\rho R_{c o}\right)-\tilde{\Omega}_{1} Y_{0}\left(\rho R_{c o}\right)} \\
& +\sum_{k=1}^{\infty} \tilde{A}_{k}^{(3 q)} \frac{J_{\lambda_{c b}, k}(\rho r)-\tilde{\Omega}_{k} Y_{\lambda_{c b, k}}(\rho r)}{J_{\lambda_{c b}, k}\left(\rho R_{c o}\right)-\tilde{\Omega}_{k} Y_{\lambda_{c b b}}\left(\rho R_{c o}\right)} \cos \left[\lambda_{c b, k}\left(\theta-\theta_{q}\right)\right]
\end{aligned}
$$

where $\tilde{A}_{0}^{(3 q)}$ and $\tilde{A}_{k}^{(3 q)}$ are the undetermined constant coefficients, which can be determined by

$$
\begin{aligned}
\tilde{A}_{0}^{(3 q)} & =\frac{1}{\theta_{c}} \int_{\theta_{q}}^{\theta_{q}+\theta_{c}} \tilde{A}_{2, m}\left(R_{c o}, \theta\right) d \theta \\
& =\tilde{A}_{0}^{(2)}+\sum_{n}\left(\begin{array}{l}
M_{q \times n}^{(8)} \tilde{A}_{n}^{(2)}-M_{q \times n}^{(9)} \tilde{B}_{n}^{(2)} \\
+M_{q \times n}^{(10)} \tilde{C}_{n}^{(2)}-M_{q \times n}^{(11)} \tilde{D}_{n}^{(2)}
\end{array}\right) \\
\tilde{A}_{k}^{(3 q)} & =\frac{2}{\theta_{c}} \int_{\theta_{q}}^{\theta_{q}+\theta_{c}} \tilde{A}_{2, m}\left(R_{c o}, \theta\right) \cos \left[\lambda_{c b, k}\left(\theta-\theta_{q}\right)\right] d \theta \\
& =\sum_{n}\left(\begin{array}{l}
M_{q \times n}^{(12)} \tilde{A}_{n}^{(2)}-M_{q \times n}^{(13)} \tilde{B}_{n}^{(2)} \\
+M_{q \times n}^{(14)} \tilde{C}_{n}^{(2)}-M_{q \times n}^{(15)} \tilde{D}_{n}^{(2)}
\end{array}\right)
\end{aligned}
$$

C. Interface conditions in region 2

By applying the interface conditions, the vector potential distributions in regions 1 and $3 q$ are determined based on the vector potential distribution in region 2. Since the PM magnetic field and the eddy current reaction field are coupled in region 2 , the expression for the vector potential in region 2 can be determined by applying the following interface conditions

$$
\begin{aligned}
& \left.\frac{\partial \tilde{A}_{1, m, t}}{\partial r}\right|_{r=R_{m i}}=\left.\frac{\partial \tilde{A}_{2, m, t}}{\partial r}\right|_{r=R_{m i}} \\
& \left.\frac{\partial \tilde{A}_{2, m, t}}{\partial r}\right|_{r=R_{c o}}=\left.\frac{\partial \tilde{A}_{3 q, m, t}}{\partial r}\right|_{r=R_{c o}} \text { with } \theta \in\left(\theta_{q}, \theta_{q}+\theta_{c}\right) .
\end{aligned}
$$

Thus, (31) can be developed as

$$
\begin{aligned}
\tilde{A}_{2, m}=\tilde{A}_{0}^{(2)}+\tilde{B}_{0}^{(2)} R_{m i} \ln r \\
+\sum_{n=1}^{\infty}\left\{\begin{array}{l}
{\left[\tilde{A}_{n}^{(2)} \frac{R_{m i}}{n} \frac{U_{r, R_{c o}}^{(n)}}{V_{R_{m i}, R_{c o}}^{(n)}}-\tilde{B}_{n}^{(2)} \frac{R_{c o}}{n} \frac{U_{r, R_{m i}}^{(n)}}{V_{R_{m i}}^{(n)} R_{c o}}\right] \cos (n \theta)} \\
\left.+\left[\tilde{C}_{n}^{(2)} \frac{R_{m i}}{n} \frac{U_{r, R_{c o}}^{(n)}}{V_{R_{m i}, R_{c o}}^{(n)}}-\tilde{D}_{n}^{(2)} \frac{R_{c o}}{n} \frac{U_{r, R_{m i}}^{(n)}}{V_{R_{m i}, R_{c o}}^{(n)}}\right] \sin (n \theta)\right\},
\end{array}\right.
\end{aligned}
$$

where we introduce the notation [10]

$$
V_{x, y}^{(z)}=\left(\frac{x}{y}\right)^{z}-\left(\frac{y}{x}\right)^{z}
$$

and the constant coefficients can be determined by

$$
\begin{aligned}
\tilde{B}_{0}^{(2)} & =\left.\frac{1}{2 \pi} \int_{0}^{2 \pi} \frac{\partial \tilde{A}_{1, m}}{\partial r}\right|_{r=R_{m i}} d \theta \\
\tilde{A}_{n}^{(2)} & =\left.\frac{1}{\pi} \int_{0}^{2 \pi} \frac{\partial \tilde{A}_{1, m}}{\partial r}\right|_{r=R_{m i}} \cos (n \theta) d \theta \\
& =M_{n \times n}^{(3)} \tilde{A}_{n}^{(1)}+\tilde{\Gamma}_{n}^{(1)} \\
\tilde{B}_{n}^{(2)} & =\left.\frac{1}{\pi} \sum_{q=1}^{Q} \int_{\theta_{q}}^{\theta_{q}+\theta_{c}} \frac{\partial \tilde{A}_{3 q, m}}{\partial r}\right|_{r=R_{c o}} \cos (n \theta) d \theta \\
& =\sum_{q} \tilde{M}_{n \times q}^{(4)} \tilde{A}_{0}^{(3 q)}+\sum_{q} \sum_{k} \tilde{M}_{n \times q}^{(5)} A_{k}^{(3 q)} \\
\tilde{C}_{n}^{(2)} & =\left.\frac{1}{\pi} \int_{0}^{2 \pi} \frac{\partial \tilde{A}_{1, m}}{\partial r}\right|_{r=R_{m i}} \sin (n \theta) d \theta=M_{n \times n}^{(3)} \tilde{A}_{n}^{(1)}+\tilde{\Gamma}_{n}^{(2)} \\
\tilde{D}_{n}^{(2)} & =\left.\frac{1}{\pi} \sum_{q=1}^{Q} \int_{\theta_{q}}^{\theta_{q}+\theta_{c}} \frac{\partial \tilde{A}_{3 q, m}}{\partial r}\right|_{r=R_{c o}} \sin (n \theta) d \theta \\
& =\sum_{q} \tilde{M}_{n \times q}^{(6)} \tilde{A}_{0}^{(3 q)}+\sum_{q} \sum_{k} \tilde{M}_{n \times q}^{(7)} A_{k}^{(3 q)} \cdot
\end{aligned}
$$

By substituting the general solution for the vector potential of each region ((33), (42), and (47)) into the coefficient equations ((39), (40), (43), (44), (49)-(53)), linear equations are obtained, which can be written in matrix form as

$$
[A]\{X\}=\{B\},
$$

where

$$
[A]=\left(\begin{array}{cccccccccc}
-1 & 0 & 0 & 1 & 0 & 0 & 0 & 0 & 0 & 0 \\
0 & -I_{N} & 0 & 0 & M_{N \times N}^{(1)} & M_{N \times N}^{(2)} & 0 & 0 & 0 & 0 \\
0 & 0 & -I_{N} & 0 & 0 & 0 & M_{N \times N}^{(1)} & M_{N \times N}^{(2)} & 0 & 0 \\
0 & 0 & 0 & 0 & 0 & 0 & 0 & 0 & (1)_{1 \times Q} & 0 \\
0 & M_{N \times N}^{(3)} & 0 & 0 & -I_{N} & 0 & 0 & 0 & 0 & 0 \\
0 & 0 & 0 & 0 & 0 & -I_{N} & 0 & 0 & \tilde{M}_{N \times Q}^{(4)} & \tilde{M}_{N \times K Q}^{(5)} \\
0 & 0 & M_{N \times N}^{(3)} & 0 & 0 & 0 & -I_{N} & 0 & 0 & 0 \\
0 & 0 & 0 & 0 & 0 & 0 & 0 & -I_{N} & \tilde{M}_{N \times Q}^{(6)} & \tilde{M}_{N \times K Q}^{(7)} \\
0 & 0 & 0 & (1)_{Q \times 1} & M_{Q \times N}^{(8)} & M_{Q \times N}^{(9)} & M_{Q \times N}^{(1)} & M_{Q \times N}^{(1)} & -I_{Q} & 0 \\
0 & 0 & 0 & 0 & M_{Q \times N}^{(12)} & M_{Q \times N}^{(13)} & M_{Q \times N}^{(1)} & M_{Q \times N}^{(1)} & 0 & -I_{K Q}
\end{array}\right)
$$




$$
\begin{aligned}
\{X\}= & \left\{\tilde{A}_{0}^{(1)},\left(\tilde{A}_{n}^{(1)}\right)_{N},\left(\tilde{C}_{n}^{(1)}\right)_{N}, \tilde{A}_{0}^{(2)},\left(\tilde{A}_{n}^{(2)}\right)_{N},\left(\tilde{B}_{n}^{(2)}\right)_{N},\left(\tilde{C}_{n}^{(2)}\right)_{N},\right. \\
& \left.\left(\tilde{D}_{n}^{(2)}\right)_{N},\left(\tilde{A}_{0}^{(3 q)}\right)_{Q},\left(\tilde{A}_{k}^{(3 q)}\right)_{K Q}\right\}^{T} \\
\{B\}= & \left\{0,(0)_{N},(0)_{N}, 0, \tilde{\Gamma}_{N}^{(1)},(0)_{N}, \tilde{\Gamma}_{N}^{(2)},(0)_{N},(0)_{Q},(0)_{K Q}\right\}^{T} .
\end{aligned}
$$

$N$ and $K$ are the numbers of spatial harmonics, which are used in field calculations for regions 1 and 2 , respectively. The unknown coefficients in (56) can then be determined easily by solving this Cramer system. The numerical solution can be obtained using mathematical tools such as MATLAB. The detailed forms of the elements are given in the appendix.

It can be seen that by applying the interface conditions, the constant coefficients for the vector potential in regions 1,2 , and 3 are correlated directly with each other. The time harmonic order $m$ and the spatial harmonic order $n$ and $k$ appear in the coefficient expressions for each region. Based on the linear equations ((39), (40), (43), (44), (49)(53)), the proposed analytical model is strongly coupled between the three regions, thereby considering the effect of the eddy current reaction field on the air-gap field.

\subsection{Flux density distributions}

The radial and circumferential components of the flux density in each region can be obtained from the vector potential distribution by

$\tilde{B}_{r}=\sum_{m} \frac{1}{r} \frac{\partial \tilde{A}_{i, m}}{\partial \theta}$ and $\tilde{B}_{\theta}=\sum_{m}-\frac{\partial \tilde{A}_{i, m}}{\partial r}$ with $i=1,2,3 q$.

The flux density distributions in region 1 are given by

$\tilde{B}_{l r, m}=\sum_{n=1}^{\infty}\left\{\begin{array}{l}-\frac{n}{r}\left[\tilde{A}_{n}^{(1)} \frac{U_{r, R_{m o}}^{(n)}}{U_{R_{m}, R_{m o}}^{(n)}}+\eta_{m 1} \tilde{M}_{m}^{(1)} \varsigma_{n, r}+\eta_{m 2} \tilde{N}_{1} \xi_{n, r}\right] \sin (n \theta) \\ +\frac{n}{r}\left[\tilde{C}_{n}^{(1)} \frac{U_{r, R_{m}}^{(n)}}{U_{R_{m i}, R_{m o}}^{(n)}}+\eta_{m 1} \tilde{M}_{m}^{(2)} \varsigma_{n, r}+\eta_{m 2} \tilde{N}_{2} \xi_{n, r}\right] \cos (n \theta)\end{array}\right\}$

for the radial component and

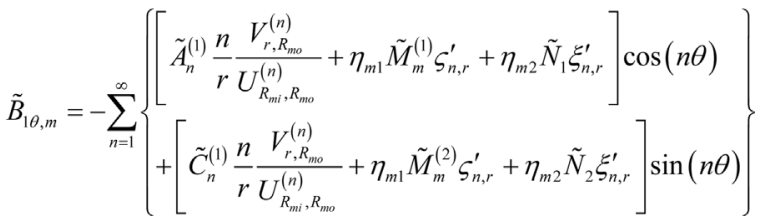

for the circumferential component, where

$$
\begin{aligned}
S_{n, r}^{\prime}= & -\frac{R_{m o}}{r}\left(\frac{R_{m o}}{R_{m i}}\right)^{n} \frac{V_{r, R_{m o}}^{(n)}}{U_{R_{m i}, R_{m o}}^{(n)}}-R_{m i} \frac{n}{r} \frac{V_{r, R_{m o}}^{(n)}}{U_{R_{m i}, R_{m o}}^{(n)}}-\left(\frac{R_{m o}}{r}\right)^{n+1}+1 \\
\xi_{r}^{\prime}= & \frac{R_{m o}}{r} \frac{R_{m o}}{R_{m i}} \frac{V_{r, R_{m o}}^{(1)}}{U_{R_{m i}}^{(1)}, R_{m o}}\left(\ln R_{m o}+1\right)-\ln R_{m i} \frac{R_{m i}}{r} \frac{V_{r, R_{m o}}^{(1)}}{U_{R_{m i}, R_{m o}}^{(1)}} \\
& +\left(\frac{R_{m o}}{r}\right)^{2}\left(\ln R_{m o}+1\right)-\ln r-1
\end{aligned}
$$

The flux density distributions in region 2 are given by

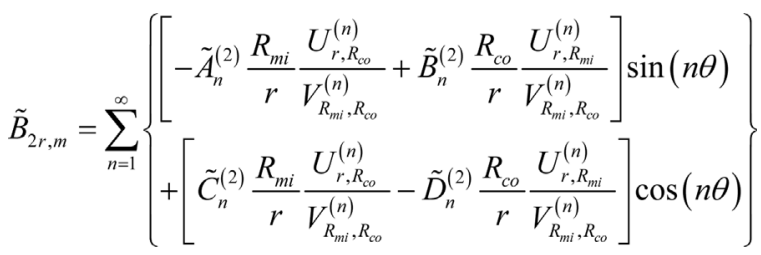

for the radial component and

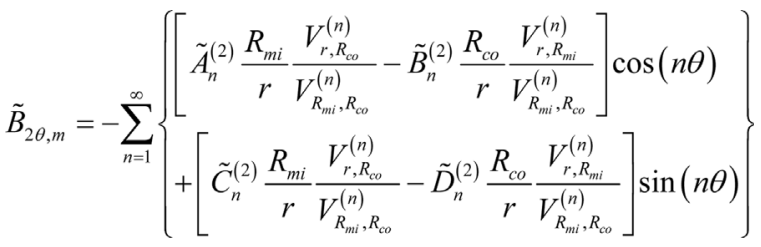

for the circumferential component.

The flux density distributions in region $3 q$ are given by

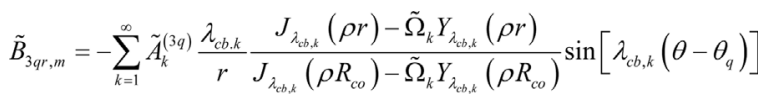

for the radial component and

$$
\begin{aligned}
& \tilde{B}_{3 g, p, m}=-\tilde{A}_{0}^{(3 q)} \frac{-\rho J_{1}(\rho r)+\tilde{\Omega}_{1} \rho Y_{1}(\rho r)}{J_{0}\left(\rho R_{\infty}\right)-\tilde{\Omega}_{1} Y_{0}\left(\rho R_{c o}\right)}
\end{aligned}
$$

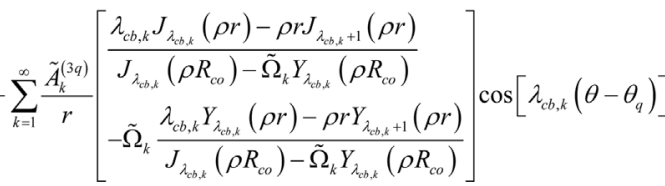

for the circumferential component.

\subsection{Electromagnetic torque}

Based on the proposed model, the electromagnetic torque of the PMEC can be obtained easily from the airgap flux density according to the Maxwell stress tensor:

$$
\begin{aligned}
T & =\sum_{m} \sum_{n} \frac{L_{a} R_{t}^{2}}{2 \mu_{0}} \int_{0}^{2 \pi} \operatorname{Re}\left(\tilde{B}_{2 r, m, n} \tilde{B}_{2 \theta, m, n}^{*}\right) d \theta \\
& =\sum_{m} \sum_{n} \frac{L_{a} \pi R_{t}^{2}}{2 \mu_{0}} \operatorname{Re}\left(-\tilde{\Psi}_{1, m, n} \tilde{\Psi}_{4, m, n}^{*}-\tilde{\Psi}_{2, m, n} \tilde{\Psi}_{3, m, n}^{*}\right),
\end{aligned}
$$

where $L_{a}$ is the axial length of the overlapping area between the magnets and the conducting bars, $R_{t}$ is the radius of the circle in the air gap, which is taken as the integration path, and the expressions of the terms are

$$
\begin{aligned}
& \tilde{\Psi}_{1, m, n}=-\tilde{A}_{n}^{(2)} \frac{R_{m i}}{R_{t}} \frac{U_{R_{t}, R_{c o}}^{(n)}}{V_{R_{m i}}^{(n)} R_{c o}}+\tilde{B}_{n}^{(2)} \frac{R_{c o}}{R_{t}} \frac{U_{R_{t}}^{(n), R_{m i}}}{V_{R_{m i}, R_{c o}}^{(n)}} \\
& \tilde{\Psi}_{2, m, n}=\tilde{C}_{n}^{(2)} \frac{R_{m i}}{R_{t}} \frac{U_{R_{t}, R_{c o}}^{(n)}}{V_{R_{m i}}^{(n)}, R_{c o}}-\tilde{D}_{n}^{(2)} \frac{R_{c o}}{R_{t}} \frac{U_{R_{t}}^{(n), R_{m i}}}{V_{R_{m i}}^{(n)} R_{c o}} \\
& \tilde{\Psi}_{3, m, n}=\tilde{A}_{n}^{(2)} \frac{R_{m i}}{R_{t}} \frac{V_{R_{t}, R_{c o}}^{(n)}}{V_{R_{m i}, R_{c o}}^{(n)}}-\tilde{B}_{n}^{(2)} \frac{R_{c o}}{R_{t}} \frac{V_{R_{t}}^{(n)}, R_{m i}}{V_{R_{m i}, R_{c o}}^{(n)}}
\end{aligned}
$$




$$
\tilde{\Psi}_{4, m, n}=\tilde{C}_{n}^{(2)} \frac{R_{m i}}{R_{t}} \frac{V_{R_{t}, R_{c o}}^{(n)}}{V_{R_{m i}, R_{c o}}^{(n)}}-\tilde{D}_{n}^{(2)} \frac{R_{c o}}{R_{t}} \frac{V_{R_{t}, R_{m i}}^{(n)}}{V_{R_{m i}, R_{c o}}^{(n)}} .
$$

\section{Model Validation}

In order to validate the proposed model, the analytical results were compared with those obtained by $\mathrm{FE}$ analysis based on 2D and three-dimensional (3D) models, using the commercial software package Ansoft Maxwell. The major parameters of the model used in the validation are given in Table 1. The permeability and conductivity of the yoke and iron-core protrusions are not considered in the analytical model, so in order to demonstrate the effectiveness and limitations of the analytical model, the nonlinear properties of the material were considered in the FE analysis. Table 2 compares the nonlinear features considered in the analytical model and the FE model. The material used for the yoke and the protrusions in the FE model was set to "steel_1008." For the sake of simplicity and generality, the validation was performed based on a simple model, which could be considered as a benchmark. The initial angular position of the PM rotor was set to $\delta_{\theta}$ $=0$.

Table 2. Nonlinear features of the model

\begin{tabular}{ccc}
\hline \hline Model & Saturation effect & Conductivity of the iron \\
\hline Analytical model & $\begin{array}{l}\text { Neglected } \\
\left(\mu_{\text {iron }} \rightarrow \infty\right)\end{array}$ & $\begin{array}{c}\text { Neglected } \\
\left(\sigma_{\text {iron }}=0 \mathrm{~S} / \mathrm{m}\right)\end{array}$ \\
2D FE model & $\begin{array}{l}\text { Considered } \\
\text { (steel_1008) }\end{array}$ & $\begin{array}{c}\text { Considered } \\
\left(\sigma_{\text {iron }}=2 \times 10^{6} \mathrm{~S} / \mathrm{m}\right)\end{array}$ \\
3D FE model & Considered & Considered \\
& $($ steel_1008) & $\left(\sigma_{\text {iron }}=2 \times 10^{6} \mathrm{~S} / \mathrm{m}\right)$ \\
\hline
\end{tabular}

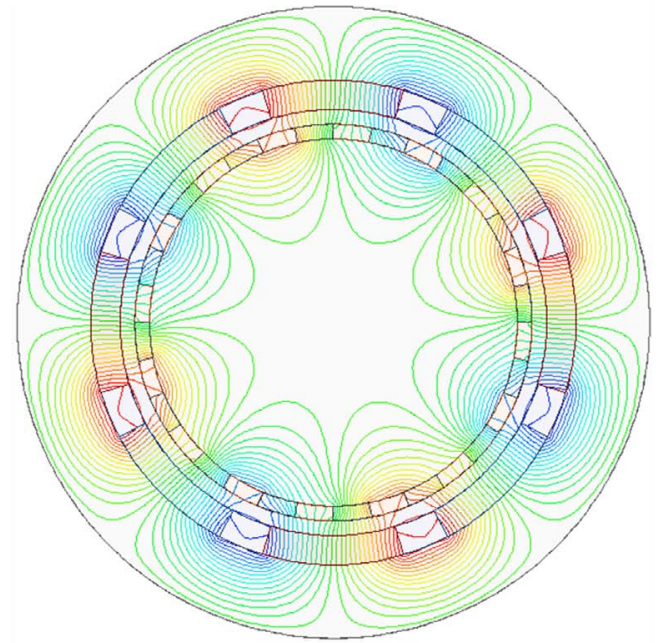

(a)

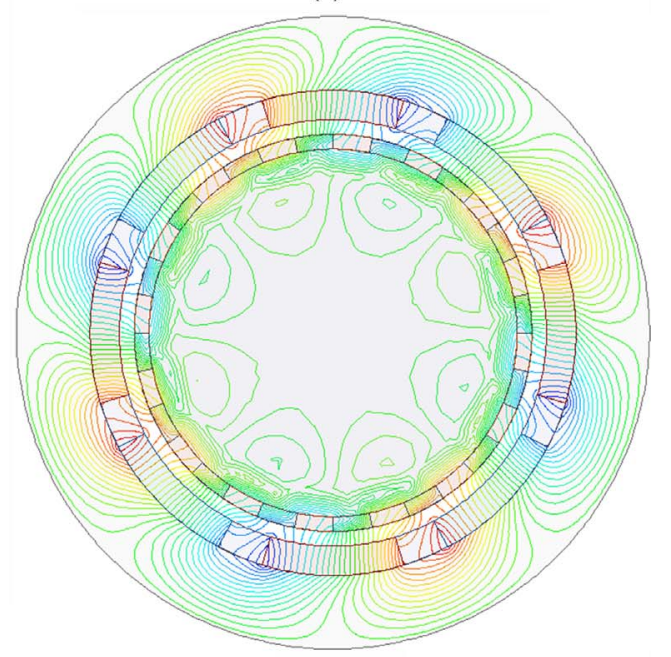

(b)

Fig. 3. (Color online) Flux lines obtained from the $2 \mathrm{D}$ nonlinear FE simulation: (a) at a slip speed of $n_{1} s=20 \mathrm{rpm}$ and (b) at a slip speed of $n_{1} s=2000 \mathrm{rpm}$.

Table 1. Parameters of the model

\begin{tabular}{|c|c|c|}
\hline Parameters & Symbol & Value \\
\hline Number of pole pairs & $p$ & 4 \\
\hline Number of conducting bars & $Q$ & 16 \\
\hline Pole-arc to pole-pitch ratio & $\alpha$ & 0.75 \\
\hline Bar-arc to slot-pitch ratio & $\beta$ & 0.5 \\
\hline Outer radius of the magnets & $R_{m o}$ & $33 \mathrm{~mm}$ \\
\hline Inner radius of the magnets & $R_{m i}$ & $29 \mathrm{~mm}$ \\
\hline Outer radius of the conducting bars & $R_{c o}$ & $27 \mathrm{~mm}$ \\
\hline Inner radius of the conducting bars & $R_{c i}$ & $25 \mathrm{~mm}$ \\
\hline Axial length of the overlapping area between magnets and bars & $L_{a}$ & $40 \mathrm{~mm}$ \\
\hline Axial length of the model & $L$ & $50 \mathrm{~mm}$ \\
\hline Remanence of magnets & $B_{r}$ & $1.18 \mathrm{~T}$ \\
\hline Conductivity of conductor & $\sigma$ & $5.8 \times 10^{7} \mathrm{~S} / \mathrm{m}$ \\
\hline Number of time harmonics used for computation of time-varied field & $M(m)$ & 25 \\
\hline Number of spatial harmonics used for magnetic field computation in region 1 and 2 & $N(n)$ & 100 \\
\hline Number of spatial harmonics used for magnetic field computation in region $3 q$ & $K(k)$ & 5 \\
\hline
\end{tabular}


Figure 3 shows the flux lines in the 2D FE model with a low value $(20 \mathrm{rpm})$ and a high value $(2000 \mathrm{rpm})$ for the slip speed, respectively. The corresponding circumferential and radial components of the flux density distribution in regions 2 and $3 q$ calculated from (63)-(66) are shown in Figs. 4-7. The value of the slip speed was selected arbitrarily to validate the analytical model. The components calculated in region 2 (at $r=27.5 \mathrm{~mm}$ ) and region 3 (slot 1 and slot 2, at $r=26 \mathrm{~mm}$ ) were compared with the results obtained from the FE simulation.

Figures. 4 and 6 show that the analytical predictions obtained by the proposed model were in excellent agreement with the FE results for the flux density distributions in the air gap and conducting bars at a low slip speed. At a high slip speed, as shown in Figs. 5 and 7, the analytical results had high accuracy according to the 2D FE prediction, but they differed slightly from the 3D FE results. It is interesting to note that the main reason for this discrepancy was due to model assumptions (3) and (5). In fact, in the analytical calculation and 2D FE analysis, at both ends of the magnet along the axial direction, the flux density distribution and flux leakage (axial end effect of the magnet) were neglected, and the end path connecting the bars was treated as shortened. By contrast, in the 3D

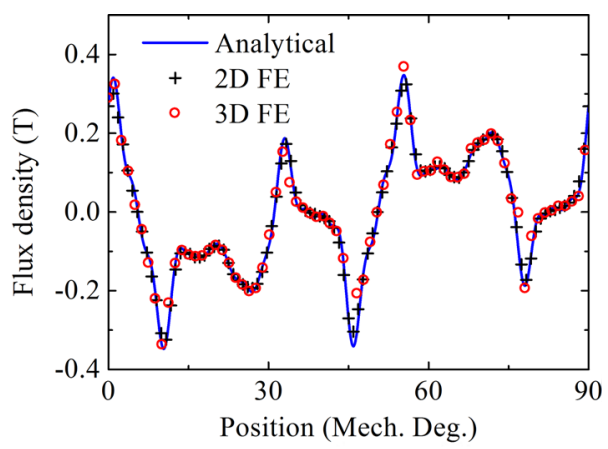

(a)

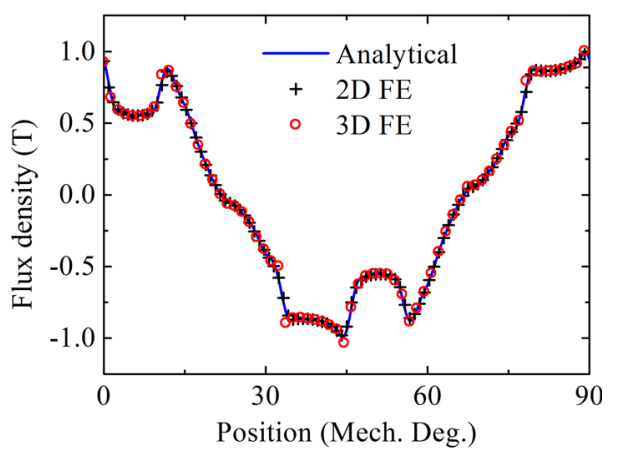

(b)

Fig. 4. (Color online) Comparison of the flux density distributions in the air gap predicted analytically and by nonlinear FE analysis at $r=27.5 \mathrm{~mm}$, with a slip speed of $n_{1} s=20 \mathrm{rpm}$ : (a) circumferential component and (b) radial component.

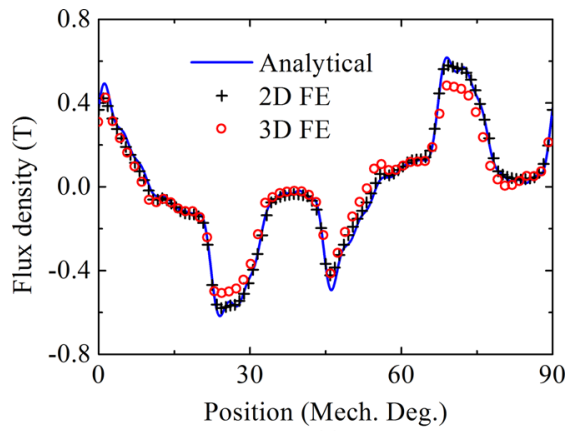

(a)

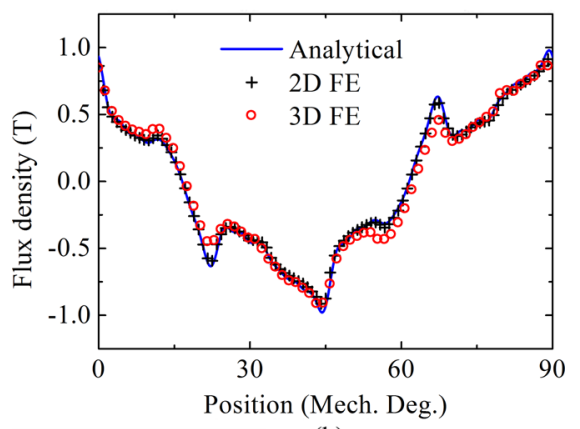

(b)

Fig. 5. (Color online) Comparison of the flux density distributions in the air gap predicted analytically and by nonlinear FE analysis at $r=27.5 \mathrm{~mm}$, with a slip speed of $n_{1} s=2000$ rpm: (a) circumferential component and (b) radial component.

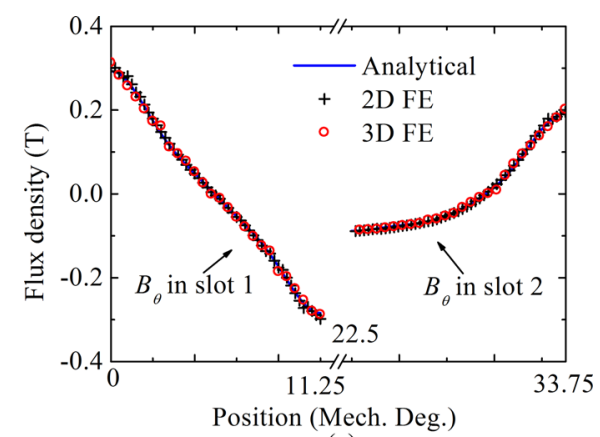

(a)

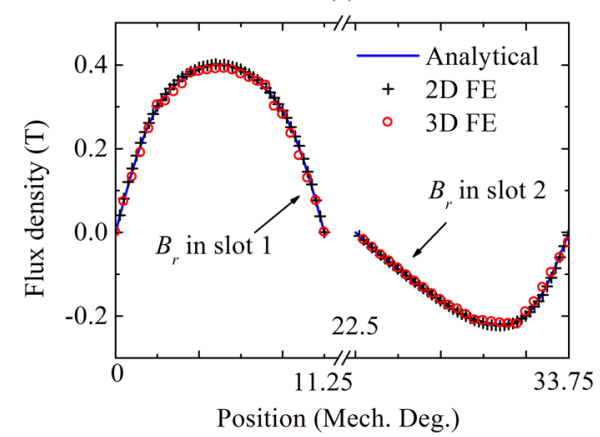

(b)

Fig. 6. (Color online) Comparison of the flux density distributions in the conducting bars ( $q=1$ and 2) predicted analytically and by nonlinear FE analysis at $r=27.5 \mathrm{~mm}$, with a slip speed of $n_{1} s=20 \mathrm{rpm}$ : (a) circumferential component and (b) radial component. 


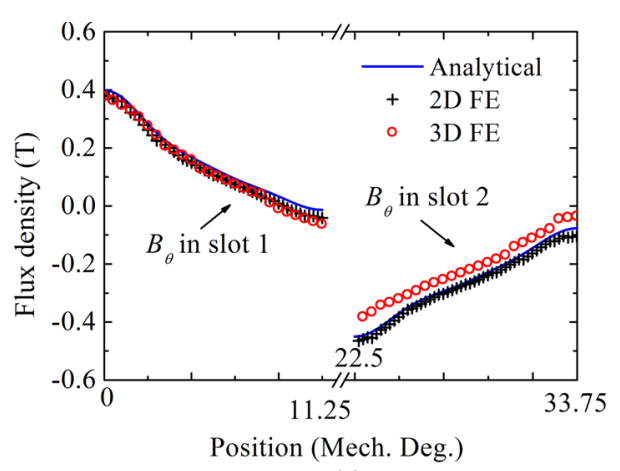

(a)

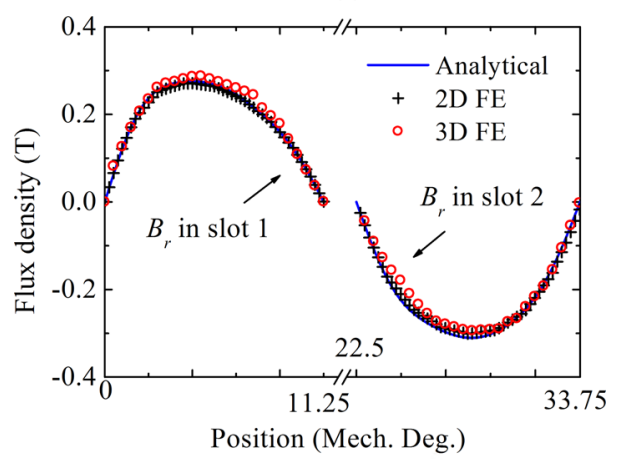

(b)

Fig. 7. (Color online) Comparison of the flux density distributions in the conducting bars $(q=1$ and 2$)$ predicted analytically and by nonlinear FE analysis at $r=27.5 \mathrm{~mm}$, with a slip speed of $n_{1} s=2000 \mathrm{rpm}$ : (a) circumferential component and (b) radial component.

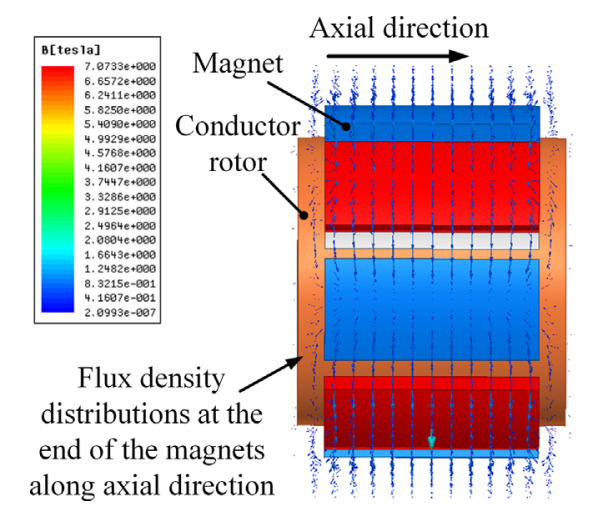

Fig. 8. (Color online) Axial end effect of the magnets.

FE model, both the end effect and the real eddy current path at the end ring of the conductor rotor were considered. Figure 8 shows the axial end effect calculated by the 3D FE simulation, where the back iron of the magnet rotor is hidden in this figure to show the flux density distribution more clearly. It can be seen that because the magnets had a finite axial length in the real model, the flux density distribution at the both ends of the magnet along the axial direction could be studied in the $3 \mathrm{D} F E$

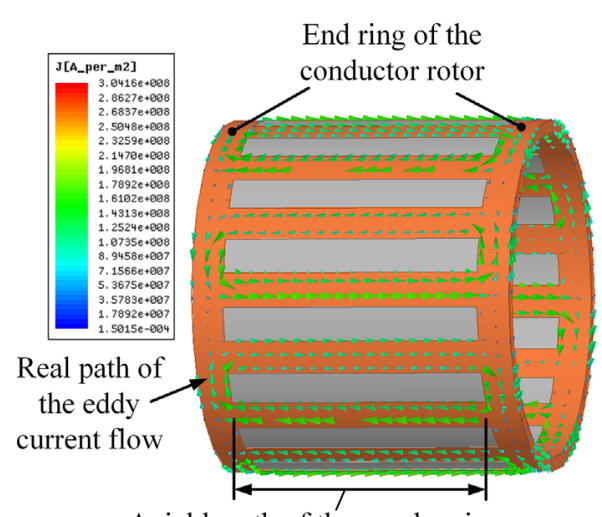

Axial length of the overlapping area between the conducting bars and magnets

Fig. 9. (Color online) Real eddy current path of the conductor rotor.

model. Figure 9 shows the real eddy current path at the conductor rotor obtained from the 3D FE simulation, which indicates that in the real model, the eddy currents flowed through the end ring and bars to form a closed loop and this could be analyzed correctly in the $3 \mathrm{D}$ FE model. However, it should be noted that when the axial length of the overlapping area between the magnets and bars relative to the axial length of the whole model had a larger ratio, the influence of these effects on the magnetic field was smaller. In practical applications, the large axial length of the overlapping area between the magnets and bars are often adopted to improve the torque density of the device. Moreover, a comparison of the analytical results and the $2 \mathrm{D} \mathrm{FE}$ results showed that although the saturation effect and the eddy currents induced in the iron cores were both neglected in the analytical modeling, they had little effect on the accuracy of the field prediction, especially for the model with a high value of $\beta$. Similar investigations conducted with other $\beta$ parameters showed that the effects of the eddy currents induced in the protrusions only became evident when the value of $\beta$ was low $(\beta \leq 0.3)$. However, the torque density in the model decreased significantly in this range for $\beta$, so this range is not likely to be investigated in practical applications. In addition, the iron yoke of the conductor rotor could be a laminated one to diminish the eddy currents induced in the protrusions. Furthermore, the normal working range area for a PMEC corresponds to low slip values [5], so the effects of these physical phenomena are not significant.

Figure 10 compares the analytical predictions of the torque-slip-speed characteristics with the 2D and 3D FE results, which shows that the analytical results were in good agreement with the 2D FE predictions, and they were similar to those obtained by the $3 \mathrm{D}$ FE analysis. The differences between the torque predictions obtained from 


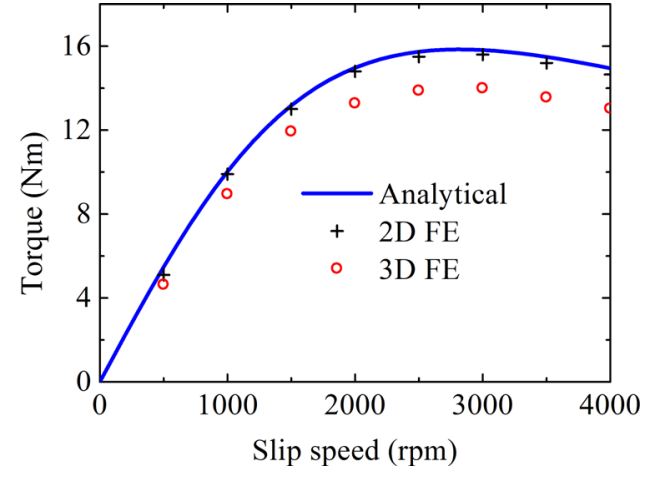

Fig. 10. (Color online) Comparison of the torque waveforms predicted analytically and by FE analysis.

the analytical model and the 3D FE analysis were due to the axial end effect, as discussed previously. Moreover, Fig. 10 shows that the waveforms of the torque calculated by the analytical and 3D FE methods followed the same trend, which means that the analytical model can be an alternative approach for studying performance and for multi-parameter optimization.

\section{Conclusion}

In this study, we proposed a comprehensive and accurate analytical model for predicting the magnetic field distributions for a radial-flux PMEC with a slotted topology. The analytical model can be used for slotted PMECs with any slot-pole combination. The analytical model considers the complex boundary conditions caused by the slots and iron-core protrusions, the spatial harmonics caused by the slotting effects, and the influence of eddy currents on the air-gap field. We verified the validity of the proposed model by comparing the results obtained with those produced by nonlinear FE analysis.

Although the magnetic field distributions can be predicted by FE simulations, the results obtained using the proposed analytical model are meaningful and they may be valuable in the first design stage and for multi-parameter optimization given the lower computational time requirements and the production of better insights into physical phenomena.

\section{Appendix I}

The expressions for the elements in the linear equations are given as follows.

In equations (39) and (40), we have

$$
M_{N \times N}^{(1)}=\frac{R_{m i}}{n} \frac{U_{R_{m i}, R_{c o}}^{(n)}}{V_{R_{m i}, R_{c o}}^{(n)}}
$$

$$
M_{N \times N}^{(2)}=-\frac{R_{c o}}{n} \frac{2}{V_{R_{m i}, R_{c o}}^{(n)}} .
$$

In equations (50)-(53), we have

$$
\begin{aligned}
& M_{N \times N}^{(3)}=\frac{n}{R_{m i}} \frac{V_{R_{m i}, R_{m o}}^{(n)}}{U_{R_{m i}, R_{m o}}^{(n)}} \\
& \tilde{M}_{N \times N}^{(4)}=\frac{1}{\pi} \frac{-\rho J_{1}\left(\rho R_{c o}\right)+\tilde{\Omega}_{1} \rho Y_{1}\left(\rho R_{c o}\right)}{J_{0}\left(\rho R_{c o}\right)-\tilde{\Omega}_{1} Y_{0}\left(\rho R_{c o}\right)} E_{q, n} \\
& \tilde{M}_{N \times N}^{(5)}=\frac{P_{q, n, k}}{\pi R_{c o}}\left[\begin{array}{l}
\frac{\lambda_{c b, k} J_{\lambda_{c b, k}}\left(\rho R_{c o}\right)-R_{c o} \rho J_{\lambda_{c b}+k}\left(\rho R_{c o}\right)}{J_{\lambda_{c b, k}}\left(\rho R_{c o}\right)-\tilde{\Omega}_{k} Y_{\lambda_{c b}, k}\left(\rho R_{c o}\right)} \\
-\tilde{\Omega}_{k} \frac{\lambda_{c b, k} Y_{\lambda_{c b}, k}\left(\rho R_{c o}\right)-R_{c o} \rho Y_{\lambda_{c b}, k+1}\left(\rho R_{c o}\right)}{J_{\lambda_{c b}, k}\left(\rho R_{c o}\right)-\tilde{\Omega}_{k} Y_{\lambda_{c b}, k}\left(\rho R_{c o}\right)}
\end{array}\right] \\
& \tilde{M}_{N \times N}^{(6)}=\frac{1}{\pi} \frac{-\rho J_{1}\left(\rho R_{c o}\right)+\tilde{\Omega}_{1} \rho Y_{1}\left(\rho R_{c o}\right)}{J_{0}\left(\rho R_{c o}\right)-\tilde{\Omega}_{1} Y_{0}\left(\rho R_{c o}\right)} F_{q, n} \\
& \tilde{M}_{N \times N}^{(7)}=\frac{Q_{q, n, k}}{\pi R_{c o}}\left[\begin{array}{l}
\frac{\lambda_{c b, k} J_{\lambda_{c b}, k}\left(\rho R_{c o}\right)-R_{c o} \rho J_{\lambda_{c b b, k}+1}\left(\rho R_{c o}\right)}{J_{\lambda_{c b}, k}\left(\rho R_{c o}\right)-\tilde{\Omega}_{k} Y_{\lambda_{c b}, k}\left(\rho R_{c o}\right)} \\
-\tilde{\Omega}_{k} \frac{\lambda_{c b, k} Y_{\lambda_{c b}, k}\left(\rho R_{c o}\right)-R_{c o} \rho Y_{\lambda_{c b, k}+1}\left(\rho R_{c o}\right)}{J_{\lambda_{c b}, k}\left(\rho R_{c o}\right)-\tilde{\Omega}_{k} Y_{\lambda_{c b, k}}\left(\rho R_{c o}\right)}
\end{array}\right] \\
& \tilde{\Gamma}_{N}^{(1)}=\eta_{m 1} \tilde{M}_{m}^{(1)}\left[\left(\frac{R_{m o}}{R_{m i}}\right)^{n+1} \frac{V_{R_{m i}, R_{m o}}^{(n)}}{U_{R_{m i}, R_{m o}}^{(n)}}+n \frac{V_{R_{m i}, R_{m o}}^{(n)}}{U_{R_{m i}, R_{m o}}^{(n)}}+\left(\frac{R_{m o}}{R_{m i}}\right)^{n+1}-1\right] \\
& -\eta_{m 2} \tilde{N}_{1}\left[\begin{array}{l}
\left(\frac{R_{m o}}{R_{m i}}\right)^{2} \frac{V_{R_{m i}}^{(1)} R_{m o}}{U_{R_{m i}}^{(1)}, R_{m o}}\left(\ln R_{m o}+1\right)+\ln R_{m i} \frac{V_{R_{m i}, R_{m o}}^{(1)}}{U_{R_{m i}, R_{m o}}^{(1)}} \\
+\left(\frac{R_{m o}}{R_{m i}}\right)^{2}\left(\ln R_{m o}+1\right)-\ln R_{m i}-1
\end{array}\right] \\
& \tilde{\Gamma}_{N}^{(2)}=\eta_{m 1} \tilde{M}_{m}^{(2)}\left[\left(\frac{R_{m o}}{R_{m i}}\right)^{n+1} \frac{V_{R_{m i}, R_{m o}}^{(n)}}{U_{R_{m i}}^{(n)}, R_{m o}}+n \frac{V_{R_{m i}, R_{m o}}^{(n)}}{U_{R_{m i}, R_{m o}}^{(n)}}+\left(\frac{R_{m o}}{R_{m i}}\right)^{n+1}-1\right. \\
& -\eta_{m 2} \tilde{N}_{2}\left[\begin{array}{l}
\left(\frac{R_{m o}}{R_{m i}}\right)^{2} \frac{V_{R_{m i}}^{(1)} R_{m o}}{U_{R_{m i}}^{(1)}\left(R_{m o}\right.}\left(\ln R_{m o}+1\right)+\ln R_{m i} \frac{V_{R_{m i}}^{(1)}, R_{m o}}{U_{R_{m i}}^{(1)}, R_{m o}} \\
+\left(\frac{R_{m o}}{R_{m i}}\right)^{2}\left(\ln R_{m o}+1\right)-\ln R_{m i}-1
\end{array}\right]
\end{aligned}
$$

where

$$
\begin{aligned}
& E_{q, n}=\int_{\theta_{q}}^{\theta_{q}+\theta_{c}} \cos (n \theta) d \theta=\frac{\sin \left[n\left(\theta_{q}+\theta_{c}\right)\right]-\sin \left(n \theta_{q}\right)}{n} \\
& F_{q, n}=\int_{\theta_{q}}^{\theta_{q}+\theta_{c}} \sin (n \theta) d \theta=\frac{\cos \left(n \theta_{q}\right)-\cos \left[n\left(\theta_{q}+\theta_{c}\right)\right]}{n} \\
& P_{q, n, k}=\int_{\theta_{q}}^{\theta_{q}+\theta_{c}} \cos \left[\lambda_{c b, k}\left(\theta-\theta_{q}\right)\right] \cos (n \theta) d \theta \\
& =\left\{\begin{array}{l}
-\frac{n}{\lambda_{c b, k}^{2}-n^{2}}\left\{(-1)^{k} \sin \left[n\left(\theta_{q}+\theta_{c}\right)\right]-\sin \left(n \theta_{q}\right)\right\}, \lambda_{c b, k} \neq n \\
\frac{\sin \left[n\left(\theta_{q}+2 \theta_{c}\right)\right]-\sin \left(n \theta_{q}\right)}{4 \lambda_{c b, k}}+\frac{\theta_{c}}{2} \cos \left(n \theta_{q}\right), \lambda_{c b, k}=n
\end{array}\right.
\end{aligned}
$$




$$
\begin{aligned}
& Q_{q, n, k}=\int_{\theta_{q}}^{\theta_{q}+\theta_{c}} \cos \left[\lambda_{c b, k}\left(\theta-\theta_{q}\right)\right] \sin (n \theta) d \theta \\
& =\left\{\begin{array}{l}
\frac{n}{\lambda_{c b, k}^{2}-n^{2}}\left\{(-1)^{k} \cos \left[n\left(\theta_{q}+\theta_{c}\right)\right]-\cos \left(n \theta_{q}\right)\right\}, \lambda_{c b, k} \neq n . \\
-\frac{\cos \left[n\left(\theta_{q}+2 \theta_{c}\right)\right]-\cos \left(n \theta_{q}\right)}{4 \lambda_{c b, k}}+\frac{\theta_{c}}{2} \sin \left(n \theta_{q}\right), \lambda_{c b, k}=n
\end{array}\right.
\end{aligned}
$$

In equations (43) and (44), we have

$$
\begin{aligned}
& M_{N \times N}^{(8)}=\frac{R_{m i}}{n \theta_{c}} \frac{2}{V_{R_{m i}, R_{c o}}^{(n)}} E_{q, n} \\
& M_{N \times N}^{(9)}=-\frac{R_{c o}}{n \theta_{c}} \frac{U_{R_{c}}^{(n)}, R_{m i}}{V_{R_{m i}}^{(n), R_{c o}}} E_{q, n} \\
& M_{N \times N}^{(10)}=\frac{R_{m i}}{n \theta_{c}} \frac{2}{V_{R_{m i}, R_{c o}}^{(n)}} F_{q, n} \\
& M_{N \times N}^{(11)}=-\frac{R_{c o}}{n \theta_{c}} \frac{U_{R_{c}}^{(n)}, R_{m i}}{V_{R_{m i}}^{(n)} R_{\infty}} F_{q, n} \\
& M_{N \times N}^{(12)}=\frac{4 R_{m i}}{n \theta_{c}} \frac{1}{V_{R_{m i}, R_{\infty o}}^{(n)}} P_{q, n, k} \\
& M_{N \times N}^{(13)}=-\frac{2 R_{c o}}{n \theta_{c}} \frac{U_{R_{c}}^{(n)}, R_{m i}}{V_{R_{m i}}^{(n)}, R_{\infty \infty}} P_{q, n, k} \\
& M_{N \times N}^{(14)}=\frac{4 R_{m i}}{n \theta_{c}} \frac{1}{V_{R_{m i}, R_{c o}}^{(n)}} Q_{q, n, k} \\
& M_{N \times N}^{(15)}=-\frac{2 R_{c o}}{n \theta_{c}} \frac{U_{R_{c o v}}^{(n)} R_{R_{n i}}}{V_{R_{m i}, R_{\infty}}^{(n)}} Q_{q, n, k} .
\end{aligned}
$$

\section{Appendix II}

\section{List of symbols}

$\boldsymbol{A}$ : Magnetic vector potential

$A$ : Magnetic vector potential component

$B$ : Magnetic flux density component

$\tilde{A}_{j}^{(i)}-\tilde{D}_{j}^{(i)}$ : Constants, $i$ is the number of region, $j$ is the number of harmonic order

$\boldsymbol{H}$ : Magnetic field intensity vector

$\boldsymbol{M}$ : Magnetization vector

$M$ : Magnetization component

$j \quad$ : Complex operator

$J_{x}:$ Bessel function of with order $x$

$Y_{x} \quad$ : Neumann function with order $x$

$\mu_{0} \quad$ : Permeability of free space

$B_{r} \quad$ : Remanence of magnets

$\sigma \quad$ : Conductivity of conductor

$m$ : Order of time harmonic used for computation of time varied field

$n$ : Order of spatial harmonic used for computation in region 1 and 2

$k \quad$ : Order of spatial harmonic used for computation in region $3 q$ $\theta_{\delta} \quad$ : Initial angle position of the PM rotor

$p \quad$ : Number of pole pairs

$Q \quad$ : Number of conducting bars

$\alpha$ : Pole-arc to pole-pitch ratio

$\beta \quad$ : Bar-arc to slot-pitch ratio

$R_{m o}$ : Outer radius of the magnets (see Fig. 2)

$R_{m i}$ : Inner radius of the magnets (see Fig. 2)

$R_{c o}$ : Outer radius of the conducting bars (see Fig. 2)

$R_{c i}$ : Inner radius of the conducting bars (see Fig. 2)

$L_{a}$ : Axial length of the overlapping area between the magnets and bars

$n_{1} \quad$ : Input speed

$s \quad$ : Slip

\section{Superscripts}

(1) : PM region

(2) : Air-gap region

$(3 q)$ : Bar region

\section{Subscripts}

$r \quad$ : radial component

$\theta \quad$ : tangential component

\section{Acknowledgements}

This project is supported by National Natural Science Foundation of China (Grant No. 51375303).

\section{References}

[1] H. K. Razavi and M. U. Lampérth, IEEE Trans. Magn. 42, 405 (2006).

[2] Z. Mouton and M. J. Kamper, IEEE Trans. Ind. Electron. 61, 3367 (2014).

[3] A. Canova and B. Vusini, IEEE Trans. Magn. 41, 24 (2005).

[4] J. Wang, H. Y. Lin, S. H. Fang, and Y. K. Huang, IEEE Trans. Magn. 50, 8000109 (2014).

[5] T. Lubin and A. Rezzoug, IEEE Trans. Ind. Electron. 62, 2287 (2015).

[6] J. Y. Choi and S. M. Jang, J. Appl. Phys. 111, 07E712 (2012).

[7] X. Dai, J. Y. Cao, Y. J. Long, Q. H. Liang, J. Q. Mo, and S. G. Wang, Electr. Power Compon. Syst. 43, 1891 (2015).

[8] S. Mohammadi, M. Mirsalim, and S. Vaez-Zadeh, IEEE Trans. Energy Convers. 29, 224 (2014).

[9] L. J. Wu, Z. Q. Zhu, D. Staton, M. Popescu, and D. Hawkins, IEEE Trans. Magn. 48, 2138 (2012).

[10] P. Arumugam, T. Hamiti, and C. Gerada, IEEE Trans. Magn. 49, 5326 (2013).

[11] F. Dubas and A. Rahideh, IEEE Trans. Magn. 50, 6300320 (2014).

[12] A. A. Qazalbash, S. M. Sharkh, N. T. Irenji, R. G. Wills, and M. A. Abusara, IEEE Trans. Magn. 50, 7027308 (2014).

[13] A. Rahideh and T. Korakianitis, IET Electr. Power Appl. 6, 628 (2012). 